'Universidad CES, Medellín, Antioquia, Colombia. 2Unidad ECMO, Clínica Las

Condes. Santiago, Chile. ${ }^{3}$ Departamento Cardiovascular, Sección de Anestesiología Cardiovascular y Unidad ECMO,

Clínica Las Condes. Santiago, Chile.

aecario de Anestesia Cardiovascular.

${ }^{\text {b}}$ Enfermero perfusionista. 'Enfermera.

Fuente de apoyo financiero: No hubo apoyo financiero alguno. Los autores declaran no tener conflictos de interés.

Recibido el 12 de septiembre de 2016, aceptado el 14 de marzo de 2017.

Correspondencia a: Ricardo Poveda Jaramillo Universidad CES, Medellín, Antioquia, Colombia. ricardopovedamd@yahoo.com

\section{Terapia de reemplazo renal continua en el paciente con oxigenación por membrana extracorpórea: consideraciones para la combinación de ambas terapias}

\author{
RICARDO POVEDA ${ }^{1, \mathrm{a}}$, CHRISTIAN FAJARDO ${ }^{2, \mathrm{~b}}$, \\ ROCÍO AGLIATI ${ }^{2, \mathrm{c}}$, RODRIGO DÍAZ ${ }^{2,3}$
}

\section{Continuous renal replacement therapy in patients with extracorporeal membrane oxygenation}

The presence of renal dysfunction is not unusual in patients with pulmonary or cardiac dysfunction who require rescue with extracorporeal membrane oxygenation (ECMO). The complexity, implicit in the implementation of both techniques, can be overwhelming. This review aims to explain the most important aspects of continuous renal replacement therapy in a patient with extracorporeal support.

(Rev Med Chile 2018; 146: 78-90)

Key words: Acute Kidney Injury; Extracorporeal Membrane Oxygenation; Renal Replacement Therapy.
$\mathrm{E}$ 1 soporte cardiorrespiratorio mediante un sistema de circulación extracorpóreo (ECMO) se utiliza en la práctica clínica desde hace más de 30 años ${ }^{1}$, sin embargo, después de 2009, con la publicación del estudio CESAR, ha habido una expansión significativa del uso de $\mathrm{ECMO}^{1}$. Esta investigación evidenció la mejoría en la supervivencia en pacientes con síndrome de distrés respiratorio agudo (SDRA) tratados con ECMO durante la pandemia de gripe H1N1.

La falla multiorgánica del paciente crítico hace que este sea especialmente proclive a desarrollar una alteración de la función $\mathrm{renal}^{2}$. El uso de terapia de reemplazo renal continua (TRRC) en pacientes en ECMO mejora el equilibrio hídrico, resuelve desequilibrios electrolíticos, mejora el aporte calórico y reduce la necesidad de diuréticos ${ }^{3}$.

\section{Definición}

El ECMO es un sistema de apoyo temporal que reemplaza la función de pulmones y corazón para devolver la homeostasis a los otros órganos, mientras se administra la terapia modificadora de la enfermedad o se espera la resolución del compromiso cardiorrespiratorio ${ }^{1}$. Estos pacientes, por lo general, tienen compromiso multisistémico, siendo frecuente la afectación cardiaca, pulmonar y renal concomitante. Más de 75\% de los pacientes que requieren soporte con ECMO desarrollan 
injuria renal aguda (IRA) y 50\% requerirá terapia de reemplazo renal ${ }^{2}$.

\section{Tipos de ECMO}

El ECMO se recomienda para pacientes con insuficiencia respiratoria grave, potencialmente reversible que no responden a la terapia convencional. También se indica a pacientes con colapso circulatorio por disfunción miocárdica severa. El tipo de ECMO a administrar dependerá de la función cardíaca y pulmonar del paciente ${ }^{1}$. En el curso de la hipoxemia aislada aguda, la oxigenación extracorpórea se realiza en modo venovenoso (VV), en el que se extrae sangre de la vena cava inferior por una cánula insertada a través de la vena femoral. Después de la oxigenación, la sangre es devuelta a la aurícula derecha mediante una cánula de retorno, que se inserta a través de la vena yugular interna derecha (Figura 1A). En la configuración de ECMO venoarterial (VA), la sangre oxigenada se devuelve a la aorta, por la línea de retorno, introducida a través de las arterias femoral (Figura 1B), carótida (Figura 1C) o aorta ascendente (Figura 1D), otorgando soporte hemodinámico y respiratorio.

El circuito extracorpóreo consta de: bomba centrífuga, oxigenador, intercambiador de calor, y tubería. La bomba impulsa sangre por aspiración hacia el oxigenador. Hay un sensor que mide la velocidad del flujo de sangre. La velocidad de rotación de la bomba centrífuga y el flujo de sangre se ajustan en la consola principal. El oxigenador consta de una membrana hidrófoba que permite el intercambio de gas y evita las fugas de sangre. Adicionalmente, hay dos adaptadores luer lock en los puertos de entrada y de salida del oxigenador, que se utilizan para el cebado y la monitorización de la presión (Figura 2).

El ECMO VV direcciona posibles trombos formados en el circuito hacia los pulmones en lugar de la circulación sistémica o el cerebro, como podría suceder en el soporte VA. Igualmente, el ECMO VV puede mejorar el desempeño del corazón al incrementar el contenido de oxígeno

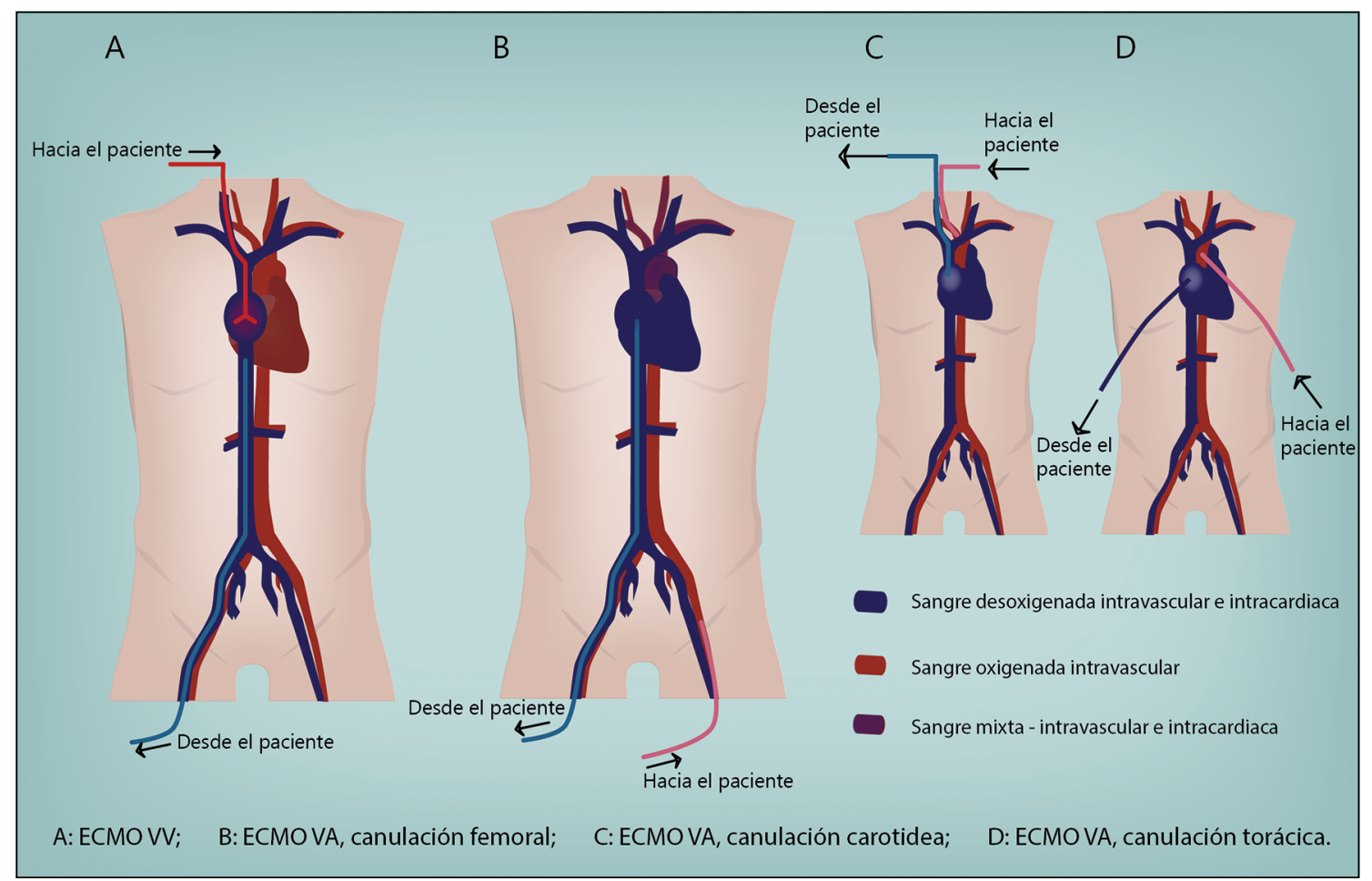

Figura 1. Tipos de ECMO. 
de la sangre venosa mixta (la sangre oxigenada es entregada en el atrio derecho directamente, no en la aorta), disminuyendo de este modo la resistencia vascular pulmonar. También evita el incremento en la postcarga del ventrículo izquierdo, mejorando la perfusión coronaria ${ }^{4}$.

Un aspecto de especial relevancia para el riñón es la conservación del flujo pulsátil en el ECMO $\mathrm{VV}$, porque, comparado con el flujo no-pulsátil del ECMO VA, disminuye la resistencia vascular y mejora la perfusión de los tejidos. La energía de la onda pulsátil mantiene abiertos los capilares y evita el reflejo neuroendocrino desencadenado por los barorreceptores en el flujo no-pulsátil y que es el responsable del aumento en la resistencia vascular periférica ${ }^{5}$. El riñón es especialmente sensible al flujo no pulsátil, haciendo necesario el uso de diuréticos para preservar el gasto urinario ${ }^{4}$.

Sin embargo, el ECMO VV no provee soporte cardíaco directo, la oferta de oxígeno puede ser inferior a la demanda, y es más dependiente de un drenaje venoso óptimo por la ineficiencia representada por la recirculación (eflujo directo de la sangre proveniente de la cánula arterial hacia la cánula venosa) $)^{4}$.

En el paciente en ECMO se emplea anticoagulación, habitualmente con heparina no frac- cionada, con infusiones entre 10 y $50 \mathrm{U} / \mathrm{kg} / \mathrm{h}$ para alcanzar mediciones de tiempo de coagulación activado entre 180 y $220 \mathrm{seg}$. La heparina se infunde directamente al circuito a través del puerto de acceso preoxigenador (Figura 2). En caso de presentarse trombocitopenia inducida por heparina, deberá suspenderse e iniciar un anticoagulante diferente, por lo general un inhibidor directo de la trombina ${ }^{6,7}$.

\section{Fisiopatología}

Los pacientes críticamente enfermos que presentan falla cardíaca aguda o disfunciones pulmonares soportados por ECMO están en alto riesgo de desarrollar IRA y sobrecarga de líquidos ${ }^{8}$, lo cual empeora su pronóstico.

A nivel molecular, tanto la enfermedad subyacente que condujo a la situación de inestabilidad del paciente, como la exposición a la superficie no endotelizada del ECMO favorecen una respuesta inflamatoria sistémica, donde los niveles de interleucinas 1,6 y 8 , y factor de necrosis tumoral alfa están elevados ${ }^{9,10}$. El estado proinflamatorio se expresa en la microcirculación generando mayor dilatación de la arteriola eferente en comparación con la aferente del glomérulo renal, resultando

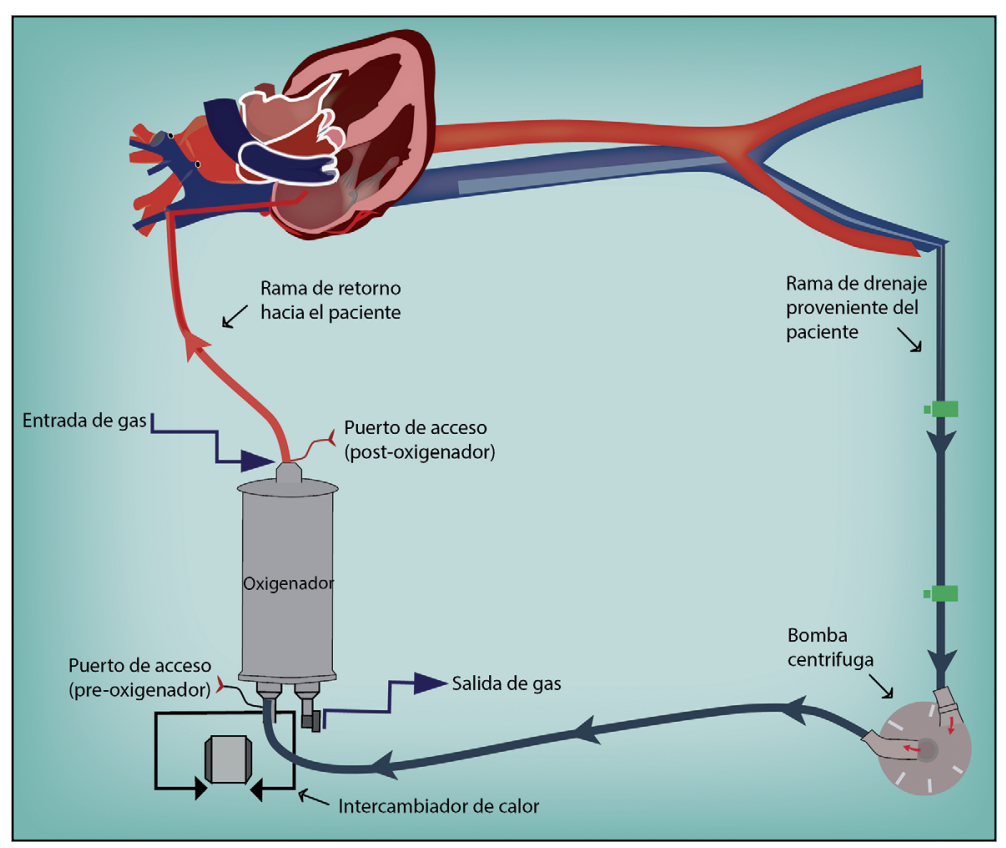

Figura 2. Componentes del ECMO. Modificado con autorización de Dr. Wojciech Szczeklik. 
en una disminución de la presión de filtración glomerular. La respuesta inflamatoria también genera disrupción del glucocálix de revestimiento en la superficie endotelial y, como consecuencia, la adhesión de los leucocitos a la pared de los vasos?.

La disfunción de bomba cardíaca que no se corrige rápidamente favorece que la insuficiencia renal progrese a necrosis cortical y daño renal permanente ${ }^{9}$. Además, la respuesta inflamatoria empeora las fugas capilares, lo que disminuye aun más el volumen intravascular'. La disminución del gasto urinario conduce a sobrecarga de líquidos que impide la oxigenación tisular y el transporte de oxígeno en los pulmones.

Otros factores involucrados en el daño renal son las especies reactivas de oxígeno y nitrógeno, el estado de hipercoagulabilidad, la hemoglobinuria, la precipitación de metahemoglobina en los túbulos renales, la exposición a agentes nefrotóxicos, y las alteraciones en la macro/microvasculatura renal $^{9}$.

Finkenstaedt y Merrill ${ }^{11}$ han atribuido la disfunción renal crónica al daño de la membrana basal de los glomérulos con regeneración epitelial anormal. Yang ${ }^{12}$, por su parte, demostró un proceso de reparación desadaptativo, con altos niveles de factor de crecimiento transformante- $\beta 1$ y factor de crecimiento del tejido conectivo, asociado a fibrosis desorganizada y abundante de los epitelios tubulares después de sufrir un episodio de IRA.

\section{Diagnóstico de IRA}

En el año 2012 se publicaron las guías KDIGO (Kidney Disease: Improving Global Outcomes) para tratar de armonizar los criterios RIFLE (Risk, Injury, Failure; Loss, End-Stage Renal Disease) y AKIN (Acute Kidney Injury Network) de IRA ${ }^{13}$ (Tabla 1). Yan $^{14}$ no encontró diferencias significativas entre las herramientas RIFLE y AKIN como predictores de mortalidad hospitalaria en pacientes sometidos a cirugía cardiaca y tratados con ECMO, concluyendo que ambas tienen buen poder discriminativo, y que cada etapa de IRA tiene una mortalidad superior a la del estadio previo.

Aunque existen marcadores moleculares (cistatina C, $\beta$-N-Acetil- $\beta$-d-glucosaminidasa, lipocalina asociada a la gelatinasa de neutrófilo) para diagnosticar precozmente IRA, la clasificación KDIGO utiliza los niveles creatinina y gasto urinario como marcadores validados en el tiempo, no solo de filtración glomerular disminuida, sino de recuperación renal ${ }^{10}$.

Durante el soporte con ECMO, las indicaciones más frecuentes para TRRC son: exceso de líquidos (43\%), prevención de sobrecarga de volumen (16\%), IRA (35\%), y alteraciones electrolíticas $(4 \%)^{15}$ (Tabla 2). No hay consenso sobre las indicaciones exactas para TRRC utilizando los criterios RIFLE/AKIN/KDIGO. El estudio de Yan $^{14}$ solo confirmó a los pacientes con la clase Failure de la clasificación RIFLE o estadio 3 de AKIN en quienes la mortalidad hospitalaria es mayor en

Tabla 1. Diagnóstico y estadios de insuficiencia renal

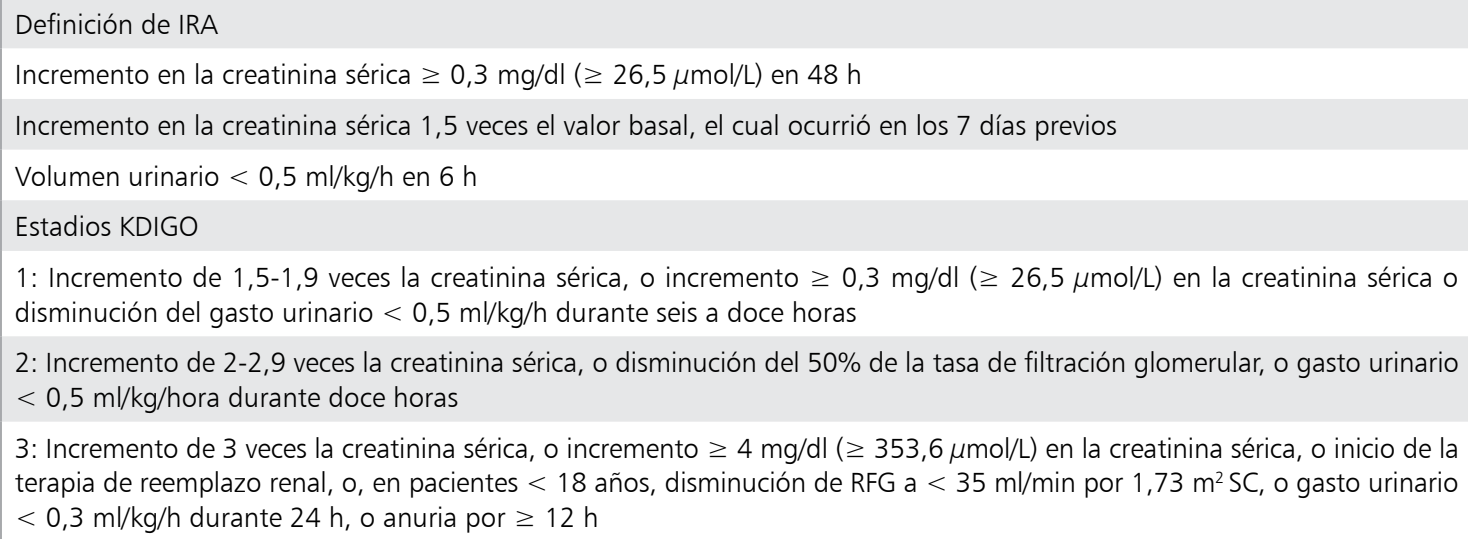

2: Incremento de 2-2,9 veces la creatinina sérica, o disminución del $50 \%$ de la tasa de filtración glomerular, o gasto urinario $<0,5 \mathrm{ml} / \mathrm{kg} /$ hora durante doce horas

3: Incremento de 3 veces la creatinina sérica, o incremento $\geq 4 \mathrm{mg} / \mathrm{dl}$ ( $\geq 353,6 \mu \mathrm{mol} / \mathrm{L}$ ) en la creatinina sérica, o inicio de la terapia de reemplazo renal, o, en pacientes $<18$ años, disminución de RFG a $<35 \mathrm{ml} / \mathrm{min}$ por 1,73 $\mathrm{m}^{2} \mathrm{SC}$, o gasto urinario $<0,3 \mathrm{ml} / \mathrm{kg} / \mathrm{h}$ durante $24 \mathrm{~h}$, o anuria por $\geq 12 \mathrm{~h}$

RFG: velocidad de filtración glomerular; SC: superficie corporal. 
Tabla 2. Indicaciones de terapia de reemplazo renal en ECMO

Oliguria o anuria menor de $200 \mathrm{ml}$ en $12 \mathrm{~h}$
Acidosis metabólica grave, con $\mathrm{pH}$ menor de 7,1
Hiperazoemia grave, con BUN igual o mayor de $80 \mathrm{mg} / \mathrm{dl}$
Hiperkalemia igual o mayor de $6,5 \mathrm{meq} / \mathrm{L}$
Signos clínicos de toxicidad urémica
Hiponatremia o hipernatremia grave, con sodio menor de
115 o mayor de 160 meq/L
Anasarca o sobrecarga de líquidos importante
Insuficiencia orgánica múltiple, incluyendo disfunción renal
SIRS, sepsis o shock séptico con disfunción renal

BUN: nitrógeno ureico en sangre; SIRS: síndrome de respuesta inflamatoria sistémica.

comparación con quienes no desarrollan IRA o quienes se clasifican en el grupo de Risk/Estadio 1.

\section{Técnicas de TRRC}

La nomenclatura del modo de la terapia de reemplazo renal se basa en el tipo de acceso vascular y en el método principal de depuración molecular. En consecuencia, se puede realizar: ultrafiltración veno-venosa continua lenta, hemofiltración veno-venosa continua, hemodiálisis veno-venosa continua y hemodiafiltración veno-venosa continua $^{16,17}$.

La hemodiálisis se basa en el principio de difusión, por el cual una alta concentración de toxinas urémicas en sangre pasan a través de una membrana semipermeable hacia una solución dializante con baja concentración de solutos. La solución dializante se mueve en contracorriente al flujo de sangre. La membrana semipermeable o filtro utilizado es usualmente de bajo flujo, y las moléculas filtradas son de pequeño tamaño (peso molecular $<500$ Dalton). La hemodiálisis provee el 10\% de la capacidad de depuración de los riñones sanos, sin embargo, no es capaz de eliminar las moléculas $>500$ Dalton ni las toxinas unidas a proteínas (Figura 3 ).

La hemofiltración depende del principio de convección: las toxinas urémicas de mayor tamaño son arrastradas y eliminadas haciendo pasar la sangre a alta presión a través de una membrana semipermeable. La presión hidrostática positiva dirige el agua a través de la membrana desde la sangre hacia el otro lado de la membrana, arrastrando con ella toxinas. Los solutos $>50.000$ Dalton no pasan a través de los poros de la membrana (la albúmina pesa 65.000 Dalton). El balance de líquidos se mantiene mediante la infusión de soluciones de recambio, que se pueden administrar antes (predilución) o después (posdilución) de la membrana. Tales soluciones se mezclan con la sangre y deben, por lo tanto, ser soluciones tamponadas, estériles, no pirogénicas y libres de endotoxinas con una composición similar a la del plasma (Figura 4).

La dosis de diálisis, en teoría, es el volumen

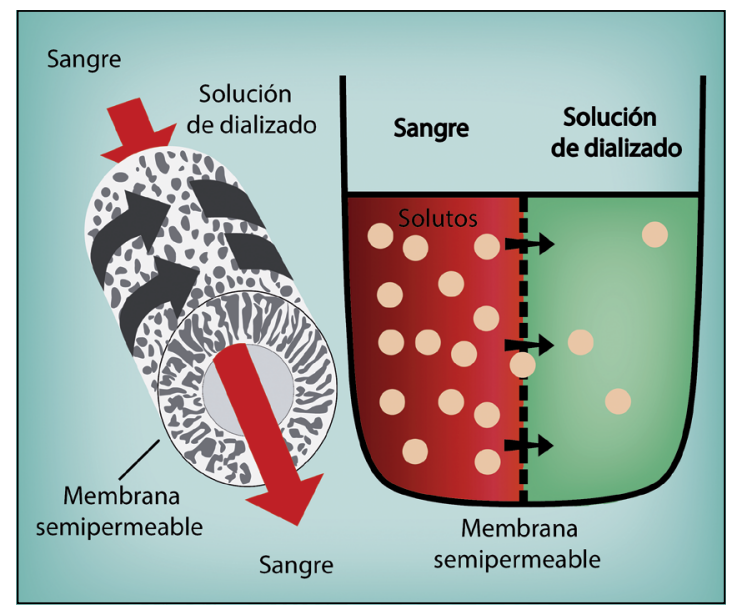

Figura 3. Principio físico de la difusión.

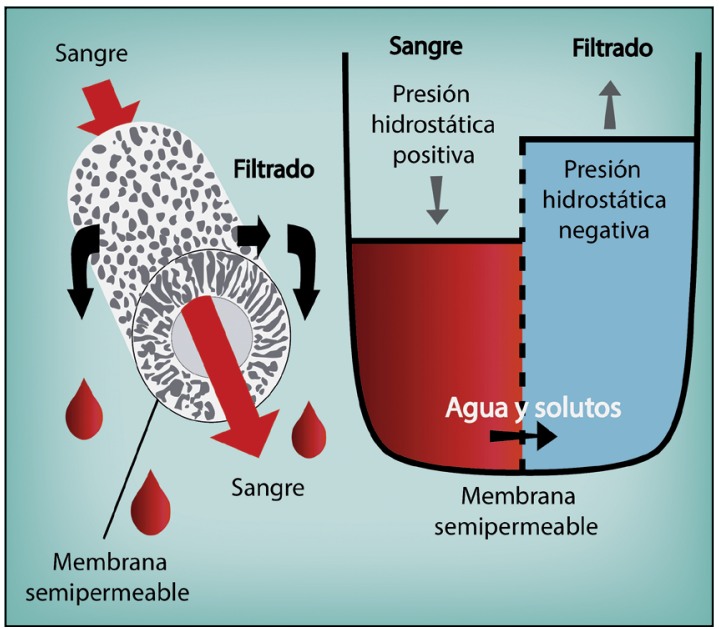

Figura 4. Principio físico de convección. 
de sangre "purificada". En la práctica, la dosis de diálisis es equivalente a la tasa de efluente en $\mathrm{ml} /$ $\mathrm{kg} / \mathrm{h}$. La tasa de efluente es la velocidad de ultrafiltración para la hemofiltración, o la suma de la velocidad de ultrafiltración y de diálisis para la hemodiafiltración. La dosis actual recomendada por KDIGO es 20 a $25 \mathrm{ml} / \mathrm{kg} / \mathrm{h}^{18}$.

La ultrafiltración continua lenta (UFCL) también esta basada en el principio de convección, no obstante, su meta principal es la extracción de bajos volúmenes de líquido ( 2 a $5 \mathrm{ml} / \mathrm{min}$, es decir, aproximadamente 3-8 1/día), por lo que no requiere la reposición de fluidos ${ }^{16}$. Es la terapia de reemplazo renal más comúnmente utilizada en línea (ver adelante) con el ECMO ${ }^{19}$. Debe subrayarse que al hacer UFCL en línea es necesario ser estricto en el control volumétrico, porque la tasa de ultrafiltración dependerá de la presión transmembrana y, en consecuencia, pueden existir variaciones entre el volumen de ultrafiltrado perseguido y el resultado final.

\section{Métodos de combinación TRRC con ECMO}

El acoplamiento de los métodos para realizar terapia de reemplazo renal continua en paciente conectado a un circuito de ECMO se puede realizar por tres vías: 1) a través de acceso venoso independiente para las dos terapias: uno para el ECMO y otro para la máquina de TRRC; 2 ) introducción de un hemofiltro en el circuito de ECMO usando bombas de infusión intravenosa para controlar el volumen ultrafiltrado y 3) con una máquina de terapia de reemplazo renal en el circuito de $\mathrm{ECMO}^{3}$.

\section{A través de acceso venoso independiente}

La forma más sencilla de realizar terapia de reemplazo renal continua es a través de un acceso venoso independiente del circuito de ECMO, sin embargo, si el paciente no tiene la canulación previa de la vía venosa central, aumenta el riesgo de complicaciones mecánicas durante procedimientos de canulación adicionales, debido a la anticoagulación en la que debe encontrarse el paciente en soporte con ECMO y la escasez de posibles accesos vasculares. Este método es más usado cuando la TRRC ya se venía haciendo en el paciente, antes de la introducción del ECMO. No obstante, hay centros que escogen esta modalidad rutinariamente ${ }^{15,17}$. En el análisis retrospectivo de Thajudeen sobre 40 pacientes, el acceso venoso separado fue una de las dos modalidades para proveer TRRC en los pacientes con $\mathrm{ECMO}^{20}$.

\section{A través de un hemofiltro en línea}

La introducción de un filtro en el circuito, o conexión en línea, es el método de TRRC más ampliamente utilizado en pacientes conectados a $\mathrm{ECMO}^{15}$. Tiene la ventaja de ser relativamente simple y barato. La línea que deriva la sangre al hemofiltro se conecta en la parte de alta presión del circuito del ECMO (postoxigenador o postbomba); la sangre vuelve proveniente del hemofiltro a la parte de baja presión del circuito (línea venosa prebomba). Debido a la existencia de un shunt, habrá una diferencia entre el flujo medido y el flujo que se suministra al paciente; tal diferencia es el volumen que se dirige hacia el hemofiltro. Se debe instalar un sensor de flujo en la vía arterial del ECMO para determinar el flujo real que se provee al paciente.

Puede requerir tres bombas de infusión externas: una conectada a la línea que emerge del hemofiltro y controla el líquido efluente, otra para proveer los líquidos de reposición y, si se requiere diálisis, otra para propulsar la solución de dializado. La técnica de TRRC que más comúnmente se emplea con el hemofiltro en línea es la ultrafiltración continua lenta ${ }^{17}$. Algunos centros utilizan esta modalidad para proporcionar sólo ultrafiltración, porque los hemofiltros están diseñados para su uso con sistemas de alta presión, haciéndolos menos efectivos para el aclaramiento difusivo $^{17}$. La técnica en línea tiene la ventaja de permitir un volumen de cebado más pequeño en comparación con el empleado cuando se suma el circuito de la máquina de TRRC. Pero también tiene desventajas: las bombas de infusión son incapaces de contener la filtración, no se monitorizan las presiones en el circuito de hemofiltración y la coagulación o la rotura del filtro no se pueden detectar tempranamente ${ }^{3}$. El control de la ultrafiltración puede ser especialmente deficiente cuando se requiere ultrafiltrar más de $800 \mathrm{ml}$ en $24 \mathrm{~h}^{17,21}$. Los datos disponibles sobre la exactitud de estos dispositivos de infusión intravenosos para regular la ultrafiltración en un circuito de ECMO describen tasas de error de hasta 12,5\%. En experimentos de laboratorio utilizando los ajustes de presión, las diferencias entre el volumen de 


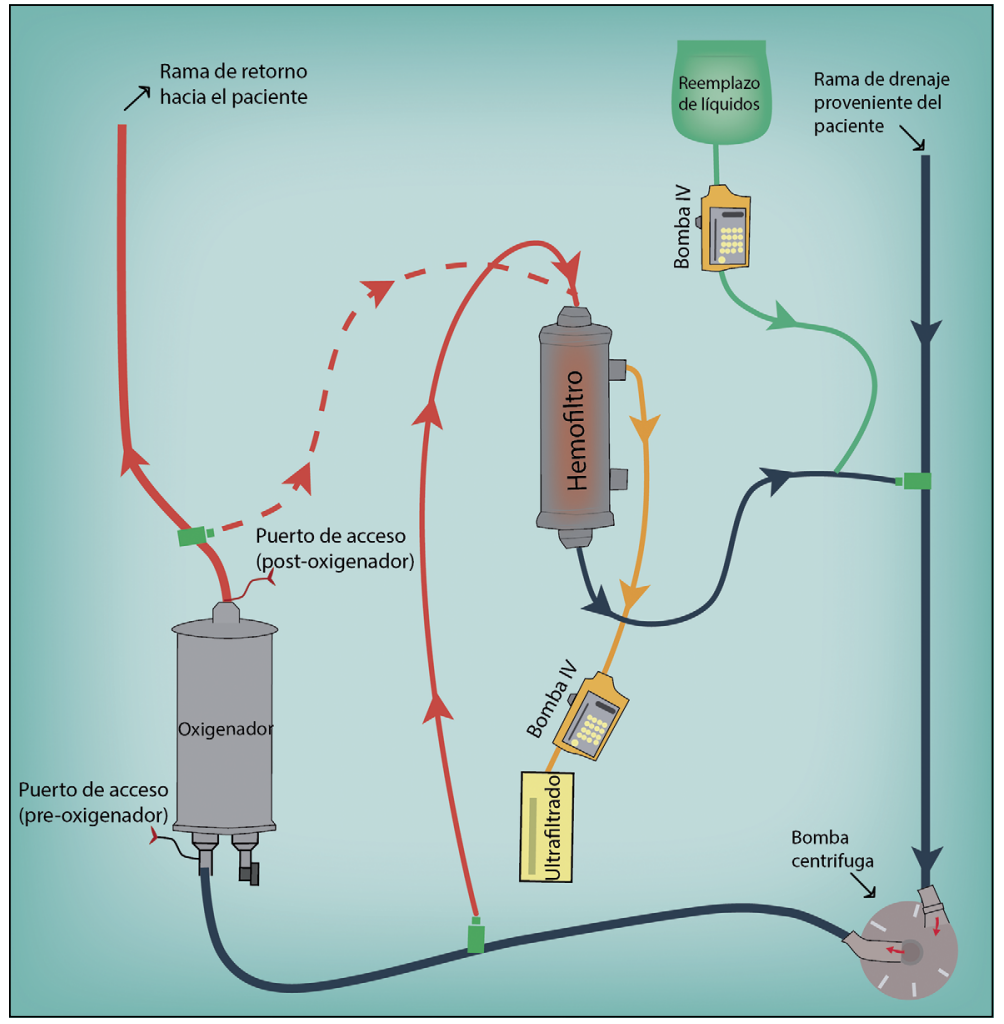

Figura 5. Hemofiltro en línea. Modificado con autorización de Dr. Wojciech Szczeklik. ultrafiltración planeado y el real fueron tan altas como $34 \mathrm{ml} / \mathrm{h}(>800 \mathrm{ml} / \mathrm{d})^{22}$. Por la disposición del hemofiltro dentro del circuito del ECMO, esta técnica genera recirculación de la sangre ya hemofiltrada, disminuyendo la eficiencia del circuito (Figura 5).

\section{A través del acoplamiento de una máquina de TRRC}

Es posible, también, conectar el circuito de una máquina de terapia de reemplazo renal al circuito de ECMO. Por lo general, la TRRC está conectada a la línea venosa del circuito de ECMO, después de la bomba centrífuga. La reconexión para devolver la sangre desde el dispositivo renal debe hacerse antes del oxigenador, para atrapar el aire o coágulos previo a su retorno al paciente (Figuras 6-10). Es un método seguro y preciso en el control del flujo de sangre en el circuito de TRRC, en el balance de fluidos y en el seguimiento de la presión transmembrana. Se pueden implementar todas las técnicas de TRRC: hemofiltración venoveno- sa continua, hemodiálisis venovenosa continua y hemodiafiltración venovenosa continua. Las diferentes formas para acoplar una máquina de TRRC al circuito del ECMO se pueden ver en la Tabla 3 y en las Figuras 5-10.

Symons observó que estos pacientes tenían un manejo más preciso de fluidos ${ }^{23}$, lo cual concuerda con los hallazgos de Chen, quien reportó que los sobrevivientes post-ECMO en TRRC tenían un mejor balance de líquidos ${ }^{15}$.

Durante la combinación de ECMO y TRRC, las alarmas de presión se pueden activar en las líneas de entrada y salida de la máquina de TRRC. Si las alarmas se activan por baja presión, deben descartarse las posibles causas: diámetro pequeño de la cánula venosa, volumen sanguíneo disminuido, obstrucción parcial por posición o una presión intratorácica negativa por esfuerzo inspiratorio del paciente. Si después de corregir la causa de la baja presión sigue activa la alarma, se debe disminuir la luz de la línea de eflujo de la máquina de TRRC, a través de una abrazadera (pinza), para aumentar 

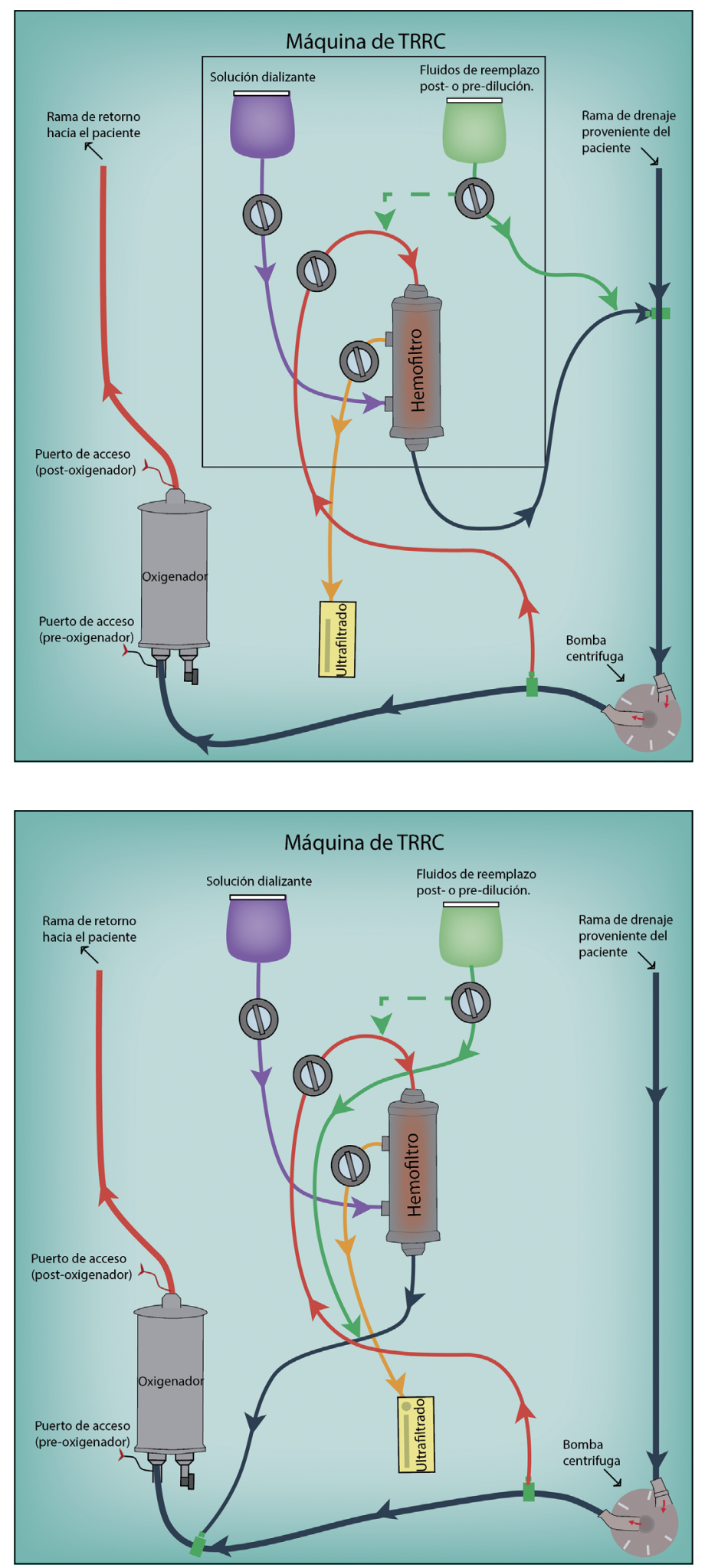

Figura 6. Modalidad Tipo I de acoplamiento del ECMO a una máquina de TRRC. Modificado con autorización de Dr. Wojciech Szczeklik.

Figura 7. Modalidad Tipo II de acoplamiento del ECMO a una máquina de TRRC. Modificado con autorización de Dr. Wojciech Szczeklik. 

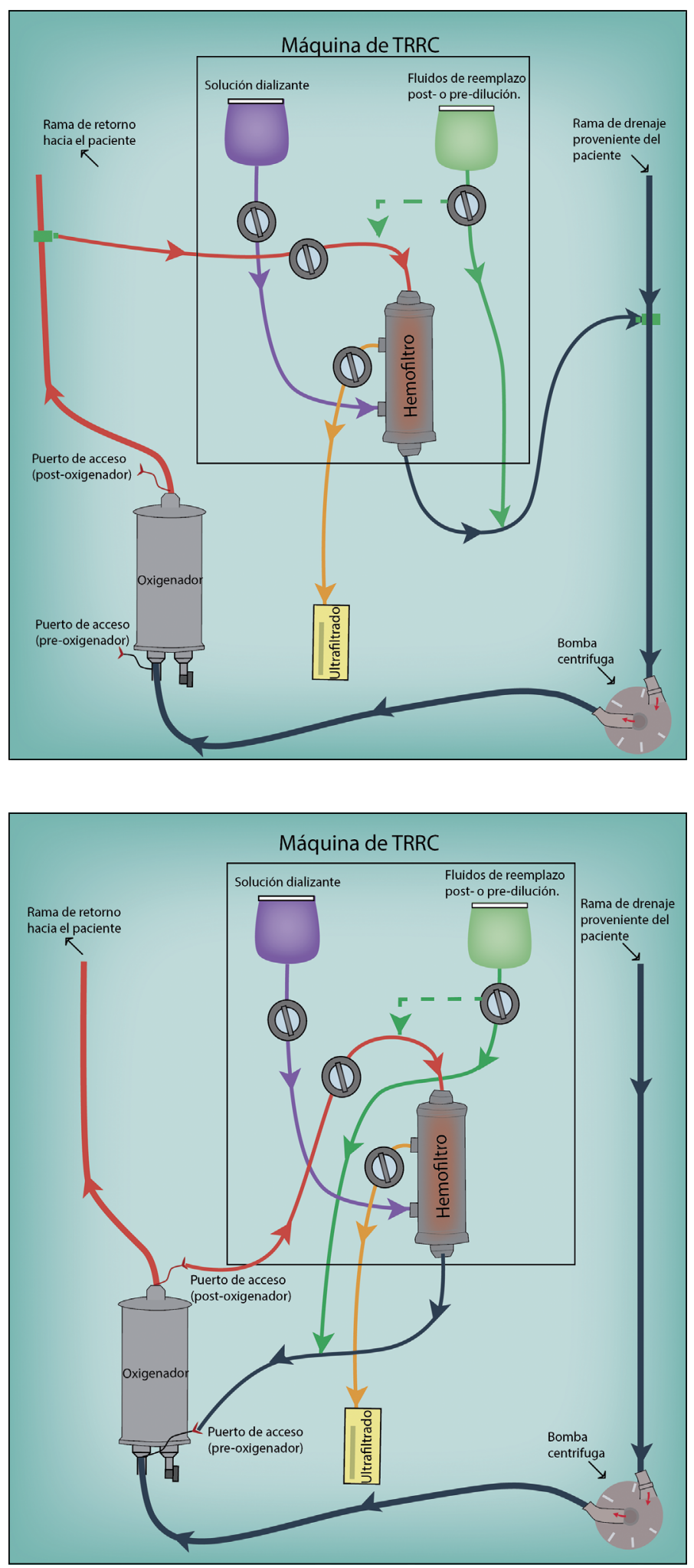

Figura 8. Modalidad Tipo III de acoplamiento del ECMO a una máquina de TRRC. Modificado con autorización de Dr. Wojciech Szczeklik.
Figura 9. Modalidad Tipo IV de acoplamiento del ECMO a una máquina de TRRC. Modificado con autorización de Dr. Wojciech Szczeklik. 


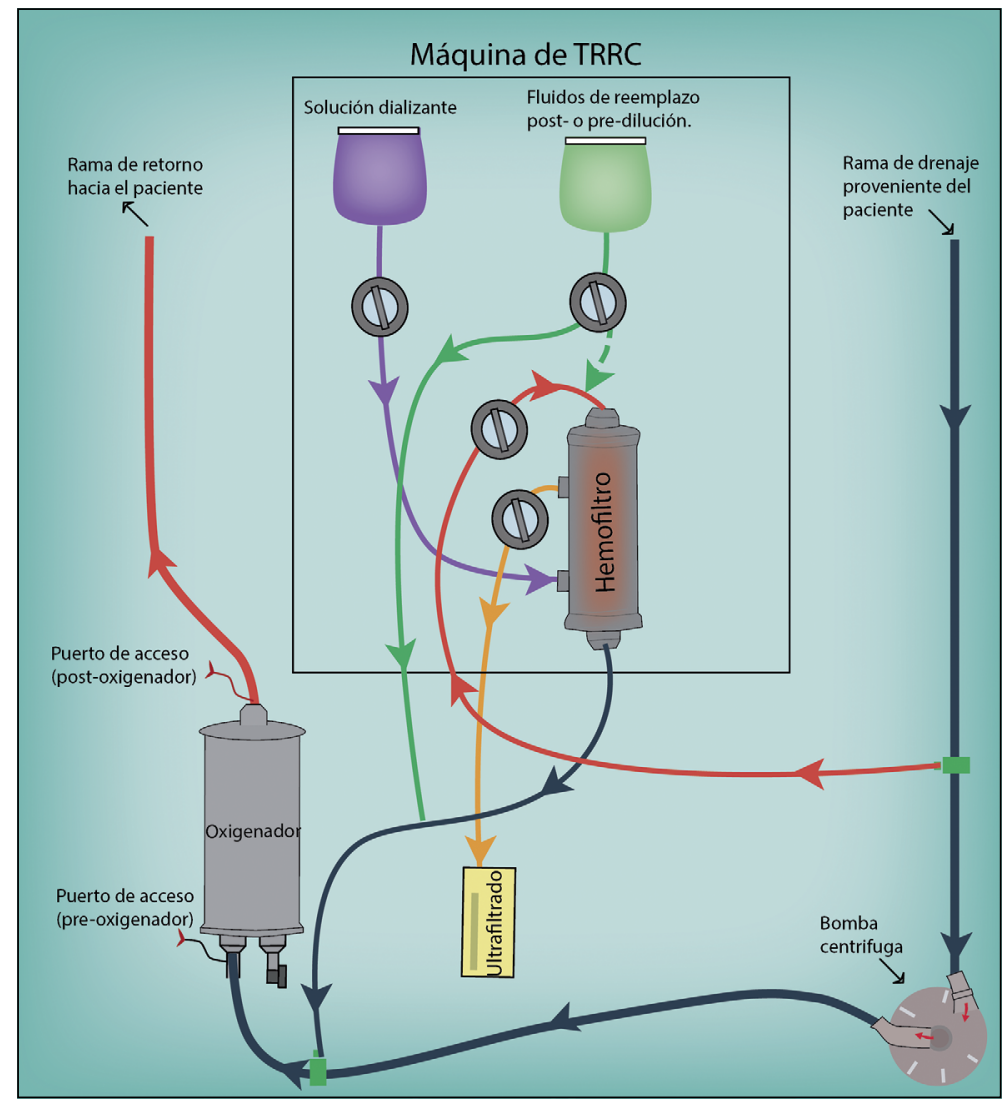

Figura 10. Modalidad Tipo $V$ de acoplamiento del ECMO a una máquina de TRRC. Modificado con autorización de Dr. Wojciech Szczeklik. la presión hidrostática; otra opción es modificar los umbrales a los que están dispuestas las alarmas del software de la máquina de TRRC ${ }^{17}$. Al igual que en la disposición de la Figura 5, los circuitos 7, 9 y 10 favorecen la recirculación.

En nuestra institución, Clínica Las Condes-Santiago de Chile, desde septiembre de 2006 hasta el presente se han realizado 220 terapias de reemplazo renal en pacientes con ECMO, entre ellas: 13 terapias de hemodiálisis, 22 terapias de hemofiltración y 97 terapias de hemodiafiltración.

\section{Pronóstico}

Tradicionalmente, se ha considerado que los pacientes sin patología renal previa, por lo general, no progresan a enfermedad renal crónica ${ }^{16}$. En el metaanálisis de Chen, cuatro de diecinueve estudios informaron la recuperación de la función renal antes del alta hospitalaria ${ }^{15}$; tres estudios mostraron una recuperación completa o ningún requerimiento de terapia de reemplazo renal en los sobrevivientes. Paden evaluó los resultados de 154 pacientes tratados con ECMO/TRRC en un centro médico pediátrico: entre 68 (44\%) sobrevivientes, la recuperación renal ocurrió en 65 (96\%) antes de su egreso del hospital ${ }^{24}$. No obstante, desde 2008 hasta 2012, múltiples estudios observacionales que evaluaron cohortes únicas demostraron que los pacientes que sobrevivena un episodio de IRA tienen un riesgo significativo de progresión a enfermedad renal crónica. Amdur ${ }^{25}$, primero y Chawla ${ }^{26}$, después, confirmaron estos hallazgos.

\section{Conclusiones}

La sobrecarga hídrica es una indicación habitual de la terapia de reemplazo renal en los pacientes con ECMO. 


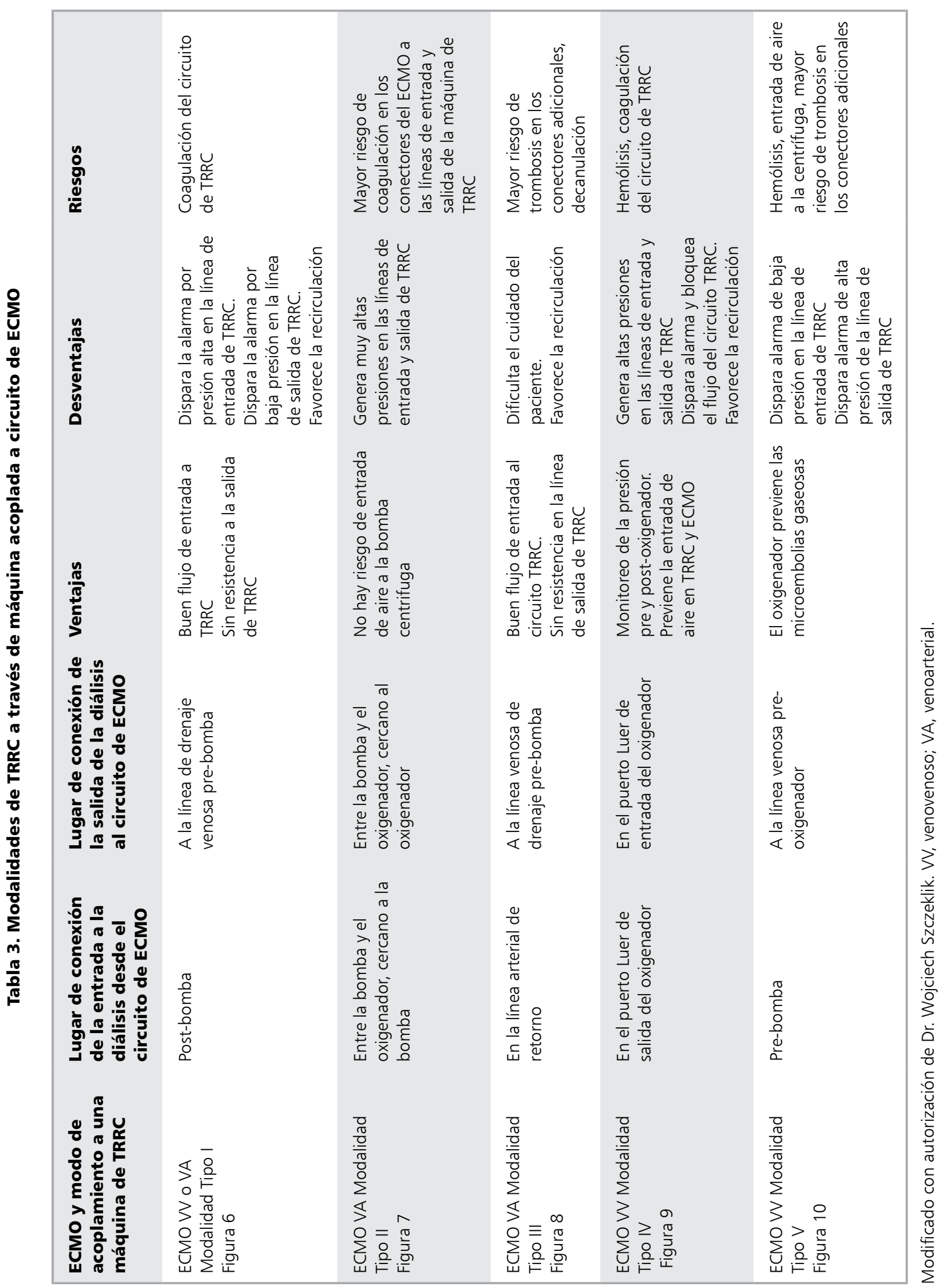


Balances hídricos positivos se asocian de forma independiente con mayor mortalidad, peor oxigenación, mayor duración de la estancia hospitalaria y mayor duración de ventilación mecánica. El fracaso para volver al peso seco también se asocia con duración prolongada del soporte con ECMO.

La introducción temprana de TRRC durante ECMO puede prevenir el exceso de líquidos y permite el tratamiento con volúmenes convencionales de farmacoterapia, sin la necesidad de utilizar altas dosis de diuréticos o restringir la ingesta hídrica.

El acoplamiento entre ECMO y TRRC se puede hacer a través de varias modalidades. Cada una con ventajas y desventajas operacionales, sin embargo, conectar una máquina de terapia de reemplazo renal al circuito de ECMO es el método más riguroso en el control del balance de fluidos.

La IRA puede causar insuficiencia renal terminal y aumentar el riesgo de desarrollar enfermedad renal crónica o empeorar la enfermedad renal crónica subyacente. En este sentido, la gravedad, duración y la frecuencia de la IRA parecen ser importantes predictores.

\section{Referencias}

1. Mosier JM, Kelsey M, Raz Y, Gunnerson KJ, Meyer R, Hypes CD, et al. Extracorporeal membrane oxygenation (ECMO) for critically ill adults in the emergency department: history, current applications, and future directions. Crit Care 2015; 17: 19: 431.

2. Chen Y-C, Tsai F-C, Fang J-T, Yang C-W. Acute kidney injury in adults receiving extracorporeal membrane oxygenation. J Formos Med Assoc 2014; 113 (11): 778-85.

3. Santiago MJ, Sánchez A, López-Herce J, Pérez R, del Castillo J, Urbano J, et al. The Use of Continuous Renal Replacement Therapy in Series with Extracorporeal Membrane Oxygenation. Kidney International 2009: 76(12): 1289-92.

4. Heard ML, Davis J, Fortenberry JD. Principles and Practice of Venovenous and Venoarterial Extracorporeal Membrane Oxygenation. In: Short BL, Williams L, eds. ECMO Specialist Training Manual 3rd edn. Ann Arbor, MI: ELSO 2012; 59-75.

5. Groom RC, Stammers AH. En: Kaplan JA, Reich DL, Savino JS, Editores, Kaplan's Cardiac Anesthesia: The Echo Era. St. Louis, Missouri, USA: Elsevier inc.; 2011. p. 921.

6. Stocker CF, Horton SB. Anticoagulation Strategies and
Difficulties in Neonatal and Paediatric Extracorporeal Membrane Oxygenation (ECMO). Perfusion 31, 2016; 2(1): 95-102.

7. Hardison DC, Fleming G. Hemofiltration and hemodialysis on ECMO. En: Short BL, Williams L, eds. ECMO Specialist Training Manual 3rd edn. Ann Arbor, MI: ELSO 2012; 189-96.

8. Zhang Z, Chen K, Ni H, Xu X. Incorporation of a hemofilter circuit into venoarterial extracorporeal membrane oxygenation: a novel approach to provide more oxygenation. Intensive Care Medicine 2015; 41 (4): 729-30.

9. Kilburn DJ, Shekar K, Fraser JF. The Complex Relationship of Extracorporeal Membrane Oxygenation and Acute Kidney Injury: Causation or Association? BioMed Research International 2016; 1-14.

10. Grocott HP, Stafford-Smith M, Mora CT. Cardiopulmonary Bypass Management and Organ Protection. En: Kaplan JA, Reich DL, Savino JS, Editores, Kaplan's Cardiac Anesthesia: The Echo Era. St. Louis, Missouri, USA: Elsevier inc.; 2011. p. 851-856.

11. Finkenstaedt JT, Merrill JP. Renal function after recovery from acute renal failure. N Engl J Med 1956; 254 : 1023-6.

12. Yang L, Besschetnova TY, Brooks CR, Shah JV, Bonventre JV. Epithelial cell cycle arrest in G2/M mediates kidney fibrosis after injury. Nat Med 2010; 16: 535-543 531p following 143.

13. Kidney Disease: Improving Global Outcomes (KDIGO) CKD Work Group. KDIGO 2012 clinical practice guideline for the evaluation and management of chronic kidney disease. Kidney Int Suppl 2013; 3 (1): 1-150.

14. Yan X, Jia S, Meng X, Dong P, Jia M, Wan J, et al. Acute kidney injury in adult postcardiotomy patients with extracorporeal membrane oxygenation: evaluation of the RIFLE classification and the Acute Kidney Injury Network criteria. Eur J Cardiothorac Surg 2010; 37 (2): 334-8.

15. Chen H, Yu RG, Yin NN, Zhou JX. Combination of extracorporeal membrane oxygenation and continuous renal replacement therapy in critically ill patients: a systematic review. Crit Care 2014; 18 (6): 675-85.

16. Karkar A. Modalities of hemodialysis: quality improvement. Saudi J Kidney Dis Transpl 2012; 23 (6): 1145-61.

17. Seczynska B, Królikowski W, Nowak I, Jankowski M, Szułdrzynski K, Szczeklik W. Continuous Renal Replacement Therapy During Extracorporeal Membrane Oxygenation in Patients Treated in Medical Intensive Care Unit: Technical Considerations. Ther Apher Dial 2014; 18(6): 523-34.

18. Kidney Disease: Improving Global Outcomes (KDIGO) CKD Work Group. KDIGO Clinical Practice Guideline 
for Acute Kidney Injury. Kidney Int Suppl 2012; 2 (1): 1-138.

19. Fleming GM, Askenazi DJ, Bridges BC, Cooper DS, Paden ML, Selewski DT, et al. A multicenter international survey of renal supportive therapy during ECMO: The Kidney Intervention During Extracorporeal Membrane Oxygenation (KIDMO) group. ASAIO J 2012; 58 (4): 407-14.

20. Thajudeen B, Kamel M, Arumugam C, Ali SA, John SG, Meister EE, et al. Outcome of patients on combined extracorporeal membrane oxygenation and continuous renal replacement therapy: a retrospective study. Int J Artif Organs 2015; 38 (3): 133-7.

21. Askenazi DJ, Selewski DT, Paden ML, Cooper DS, Bridges BC, Zappitelli M, et al. Renal replacement therapy in critically ill patients receiving extracorporeal membrane oxygenation. Clin J Am Soc Nephrol 2012; 7 (8): 1328-36.

22. Jenkins R, Chen B, Funk JE. Maximum ultrafiltration rate in continuous arteriovenous hemofiltration does not occur at the lowest level of the ultrafiltrate collection chamber. ASAIO J. 1993; 39 (3): M618-620.

23. Symons JM, McMahon MW, Karamlou T, Parrish AR, McMullan DM. Continuous renal replacement therapy with an automated monitor is superior to a free-flow system during extracorporeal life support. Pediatr Crit Care Med 2013; 14 (9): e404-408.

24. Paden ML, Warshaw BL, Heard ML, Fortenberry JD. Recovery of renal function and survival after continuous renal replacement therapy during extracorporeal membrane oxygenation. Pediatr Crit Care Med 2011; 12 (2): 153-8.
25. Amdur RL, Chawla LS, Amodeo S, Kimmel PL, Palant CE. Outcomes following diagnosis of acute renal failure in U.S. veterans: focus on acute tubular necrosis. Kidney Int 2009; 76: 1089-97.

26. Chawla LS, Amdur RL, Amodeo S, Kimmel PL, Palant $\mathrm{CE}$. The severity of acute kidney injury predicts progression to chronic kidney disease. Kidney Int 2011; 79: 1361-9.

27. Chawla LS, Kimmel PL. Acute kidney injury and chronic kidney disease: an integrated clinical syndrome. Kidney Int 2012; 82 (5): 516-24.

28. Askenazi DJ, Ambalavanan N, Hamilton K, Cutter G, Laney D, Kaslow R, et al. Acute kidney injury and renal replacement therapy independently predict mortality in neonatal and pediatric noncardiac patients on extracorporeal membrane oxygenation. Pediatr Crit Care Med 2011; 12 (1): e1-6.

29. Lin C-Y, Chen Y-C, Tsai F-C, Tian Y-C, Jenq C-C, Fang $\mathrm{J}$-T, et al. RIFLE classification is predictive of short-term prognosis in critically ill patients with acute renal failure supported by extracorporeal membrane oxygenation. Nephrol Dial Transplant 2006; 21 (10): 2867-73.

30. Nurmohamed SA, Jallah BP, Vervloet MG, Beishuizen A, Groeneveld ABJ. Predilution versus Postdilution Continuous Venovenous Hemofiltration: No Effect on Filter Life and Azotemic Control in Critically Ill Patients on Heparin: ASAIO Journal 2011; 57 (1): 48-52.

31. Macedo E, Mehta RL. Continuous Dialysis Therapies: Core Curriculum 2016. Am J Kidney Dis 2016; 68 (4): 645-57. 